\title{
Evaluación neuropsicológica de procesos cognitivos en niños de siete años de edad nacidos pretérmino
}

\author{
Montserrat Megías ${ }^{1}$, Laura Esteban ${ }^{1}$, M. Dolores Roldán-Tapia ${ }^{1}$, Ángeles F. Estévez ${ }^{1}$, \\ M. Mar Sánchez-Joya² y Julio Ramos-Lizana ${ }^{3}$ \\ 1 Departamento de Psicología, Universidad de Almería (España). \\ 2 Departamento de Enfermería, Fisioterapia y Medicina, Universidad de Almería (España). \\ 3 Servicio de Neuropediatría, Complejo Hospitalario Torrecárdenas, Almería (España).
}

\begin{abstract}
Resumen: Introducción. Diversas investigaciones han demostrado que el desarrollo cognitivo de los niños prematuros suele verse afectado, existiendo una estrecha relación entre prematuridad, déficits ejecutivos, trastornos del aprendizaje, alteraciones del lenguaje y problemas conductuales. Asimismo, destacan también las dificultades en tareas viso-perceptivas y de in tegración funcional. Objetivo. Evaluar los procesos cognitivos (funciones ejecutivas, integración viso-perceptiva, coordinación viso-motora, memoria, conocimiento del medio, lenguaje, procesamiento visomotor y aptitud motora) en niños de siete años de edad nacidos pretérmino y compararlos con un grupo control, igualado en las variables nivel sociocultural, sexo y edad. Sujetos y método. Participaron 20 niños con edades comprendidas entre siete años y dos meses y siete años y 11 meses, distribuidos homogéneamente en dos grupos (prematuro vs. control). Se elaboró un protocolo de pruebas neuropsicológicas estandarizadas para evaluar los diferentes dominios cognitivos mencionados. Resultados. Los datos obtenidos evidencian una peor ejecución en los niños prematuros en diversos dominios evaluados (v.g., funciones ejecutivas y procesamiento visomotor) en comparación con los controles. Conclusiones. Debido al escaso número de trabajos que analizan procesos cognitivos en niños nacidos pretérmino de habla hispana, en esta investigación se seleccionó la ventana de edad de 7 años, momento en el que se inician procesos esenciales -lectura, escritura y cálculo matemático-, para detectar problemas neuropsicológicos que pudiesen estar influyendo en los procesos académicos, aportando de este modo nuevos hallazgos para el ámbito de la neuropsicología infantil.

Palabras clave: Prematuridad; neuropsicología; funciones ejecutivas; dominios cognitivos.
\end{abstract}

\section{Introducción}

La comprensión del desarrollo cognitivo reclama una investigación interdisciplinar y neuropsicológica sobre la relación entre la arquitectura cerebral y el funcionamiento de los procesos cognitivos (Johnson 2000; Johnson 2003). Tomando como referente el modelo propuesto por Anderson (2002, 2008), se entiende que la actividad cognitiva está facilitada por un conjunto de funciones ejecutivas esenciales -sistema de control ejecutivo-, el cual comprende cuatro dominios distintos e interrelacionados entre sí: flexibilidad cognitiva, establecimiento de metas, control atencional y procesamiento de la información (ver Figura 1).

En este sentido, las funciones ejecutivas hacen referencia a procesos tan variados como el establecimiento de metas, la formulación de hipótesis, la organización y la planificación o secuenciación, la focalización y mantenimiento de la atención, la iniciativa, la generación de estrategias, la monitorización de la conducta, la capacidad de resolución de proble-

* Dirección para correspondencia [Correspondence address]:

Montserrat Megías. Departamento de Psicología, Universidad de Almería

(España). E-mail: mmp267@inlumine.ual.es
Title: Neuropsychological assessment of cognitive processes in seven-yearold children born prematurely.

Abstract: Introduction. Several studies have shown that the cognitive development of premature children is affected. In fact, it appears to be a close relationship between prematurity, executive deficits, learning disorders, language disorders and behavioral problems. Premature children present also difficulties in viso-perceptive tasks and functional integration tasks. $\mathrm{Ob}$ jective. The main goal of this study was to assess cognitive processes (executive functions, viso-perceptual integration, viso-motor coordination, memory, environmental knowledge, language, viso-motor processing and motor skills) in seven-year-old children who were born preterm and to compare them with those of a control group, matching in sociocultural level, sex and age. Subjects and method. 20 children ranging in age between seven years and two months and seven years and 11 months, assigned to two groups (premature vs. control) participated in the present study. A protocol including standardized neuropsychological tests was designed to assess the different cognitive mentioned domains. Results. We observed that there was worse performance in several evaluated domains (v.g., executive functions and viso-motor processing) in premature children than in the control group. Conclusions. There are few studies that explore cognitive processes in hispanic-speaking children born prematurely. In the present study we selected the seven-year age window, a time in life in which essential processes begin -reading, writing and mathematical calculation-. To detect neuropsychological problems that might be influencing the academic achievement of this population, adding new findings to the field of child neuropsychology

Key words: Prematurity; neuropsychology; executive functions; cognitive domains.

mas, la toma de decisiones, la flexibilidad cognitiva, la memoria de trabajo, la inhibición de respuestas no adecuadas o supresión de interferencias, el control y la regulación de las emociones y de la conducta. Estas funciones no sólo implican componentes de naturaleza cognitiva, sino también conductuales y emocionales, y juegan un papel esencial en la regulación de la conducta orientada a un objetivo (Elliot, 2003; Korzeniowski, 2011; Lezak, 2004; Verdejo-García y Bechara, 2010).

En un esfuerzo por hallar la relación entre determinadas áreas del cerebro y las funciones ejecutivas, diversos estudios (Houd, Rossi, Lubin y Joliot, 2010; O’Hare, Lu, Houston, Bookheimer y Sowell, 2008) han mostrado una organización jerárquica de la corteza cerebral, sugiriendo las áreas prefrontales como la base neurológica de estas funciones. Dicha relación ha sido igualmente corroborada por trabajos con poblaciones infantiles con daño cerebral, los cuales han permitido asociar daños en esta zona con ciertos déficits ejecutivos como desatención, disminución de la flexibilidad cognitiva, dificultad en la solución de problemas, limitaciones en el control de los impulsos y las emociones, o dificultades en la planificación (Stuss y Knight, 2013; Wolosin, Richardson, Hennessey, Denckla y Mostofsky, 2009). 


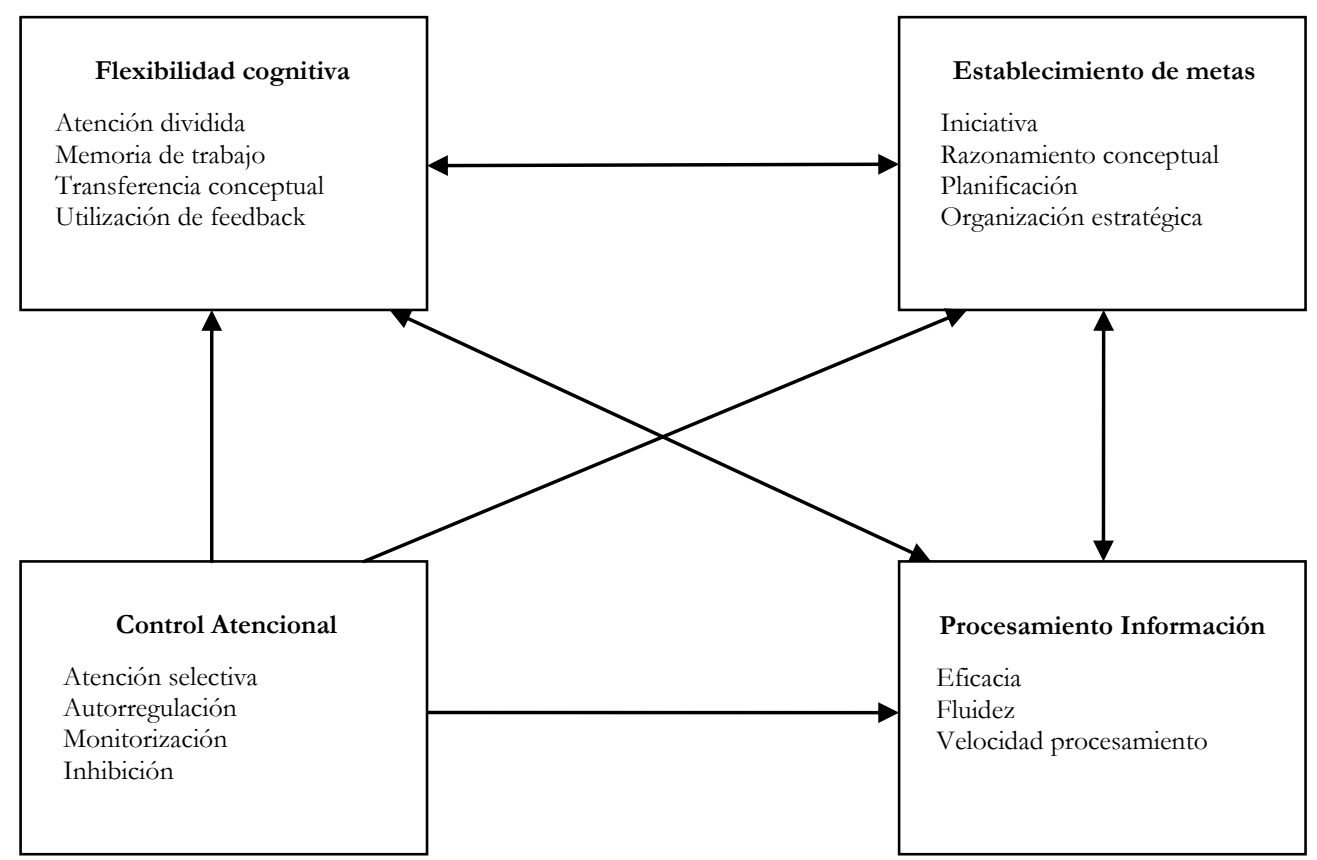

Figura 1. Sistema de Control Ejecutivo (Anderson 2002, 2008).

Con respecto a su desarrollo, autores como Cassandra y Reynolds (2005) o Portellano (2005) señalan la existencia de diferentes fases: entre 6 y 8 años las capacidades de planificación y organización se desarrollan más rápidamente, apareciendo conductas estratégicas, más organizadas y eficientes; entre 12 y 14 años se iría definiendo el control inhibitorio; mientras que otras funciones como la flexibilidad cognitiva, la memoria de trabajo o la resolución de problemas complejos seguirían desarrollándose hasta el periodo comprendido entre 15-19 años.

Como es posible corroborar, estos periodos coinciden en gran medida con las etapas de escolarización obligatoria, por lo que resulta crucial analizar procesos cognitivos en estas ventanas de edad y realizar seguimientos, a fin de detectar problemas neuropsicológicos que pudiesen estar influyendo en el rendimiento académico.

Una de las poblaciones que presentan déficits con respecto a las funciones ejecutivas, las cuales, como podemos deducir en base a los estudios revisados, son esenciales para nuestro correcto funcionamiento cotidiano, son los niños nacidos pretérmino.

En un reciente meta-análisis se ha observado que las principales áreas neuropsicológicas alteradas con respecto a las funciones ejecutivas en esta población son la memoria de trabajo, la flexibilidad y la fluidez verbal (AarnoudsseMoens, Weisglas-Kuperus, Goudoever y Oosterlaan, 2009).

Autores como Zelazo, Müeller, Frye y Marcovitch (2003) encontraron que los niños prematuros presentaban dificultades en la adquisición de habilidades básicas como el control de la atención, la planificación, la resolución y adquisición de conocimientos y la resistencia a la distracción. Estas disfunciones no son predeterminadas, sino la expresión del proceso de desarrollo (Karmiloff-Smith, 2007), ya que el control ejecutivo se elabora gradualmente desde el nacimiento (Cassandra y Reynolds, 2005; Portellano, 2005).

En consonancia con anteriores estudios sobre funciones ejecutivas y prematuridad, diversos investigadores (Pasman, Rotteveel y Maassen, 1998; Salt y Redshaw, 2006; Zelazo et al., 2003) hallaron también diferencias significativas en inhibición, memoria de trabajo y atención selectiva y mantenida durante la etapa escolar en niños nacidos pretérmino, en comparación con los nacidos a término.

Además de las dificultades en las funciones cognitivas referenciadas, en otros trabajos se ha demostrado que el desarrollo cognitivo general de los niños prematuros se encuentra afectado, existiendo una estrecha relación entre prematuridad, trastornos del aprendizaje (v.g., Briscoe, Gathercode y Marlow, 2001; Isaacs, Edmonds, Lucas y Gadian, 2001; Ment et al., 2003; O’Brien et al., 2004; Olsén et al., 1998; Pasman et al., 1998), problemas atencionales y alteraciones conductuales (Marlow, Wolke, Bracewell y Samara, 2005; Mellier, 2004; Narberhaus y Segarra, 2004; Olsén et al., 1998).

Se ha observado también que estos niños presentan en la infancia (de los 3 a los 8 años), déficit en el rendimiento cognitivo general (Burguet et al., 2000; Ment et al., 2003; Olsén et al., 1998; Peterson et al., 2000) y en ciertas habilidades no verbales (Briscoe et al., 2001).

En relación con el CI, Olsén et al. (1998) destacan las dificultades en las tareas viso-espaciales y viso-perceptivas del CI manipulativo (CIM). Autores como Briscoe et al. (2001) corroboran estas dificultades no verbales de los prematuros a través de la tarea de construcción con cubos de patrones complejos. En esta misma línea, diversos investigadores 
(Geldof, van Wassenaer, de Kieviet, Kok y Oosterlaan, 2012; Litt, Taylor, Klein y Hack, 2005; Strang-Karlsson, Andersson y Paile-Hyvarinen, 2010) hallaron que los niños muy prematuros presentaban dificultades en los procesamientos viso-espaciales y viso-motores, lo que ocasionaba un impacto negativo en los procesos de aprendizaje.

También se han observado problemas de memoria verbal, viso-espacial y de trabajo (Mathiasen, Hansen, Forman, Kessing y Greisen, 2011), así como en algunas habilidades verbales, destacando la comprensión gramatical, la comprensión auditiva de nombres de dibujos y la comprensión de órdenes verbales de complejidad creciente (Briscoe et al., 2001; Ment et al., 2003; Peterson et al., 2002).

Dado el escaso número de trabajos en los que se investigan los diferentes procesos cognitivos en niños prematuros de habla hispana en los primeros años de la infancia (6-11 años), el principal objetivo del presente estudio fue explorar dichos procesos en una etapa del desarrollo, concretamente en la ventana de edad de 7 años, en la que se inician aprendizajes tan esenciales como el de la lectura, la escritura y el cálculo matemático, tratando de averiguar qué dominios se hallan más alterados en comparación con un grupo control, a fin de detectar dificultades que puedan afectar, entre otras cosas, a su rendimiento escolar. En base a los estudios previos que acabamos de revisar, se espera encontrar una peor ejecución en algunos de los dominios cognitivos evaluados en el grupo de niños prematuros, especialmente en aquéllos relacionados con las funciones ejecutivas y con los procesamientos viso-perceptivo y viso-motor.

\section{Método}

\section{Participantes}

La muestra seleccionada para este estudio consta de 20 niños (8 niños y 12 niñas), de edades comprendidas entre siete años y dos meses y siete años y 11 meses, distribuidos homogéneamente en dos grupos: a) Grupo Experimental: 10 niños nacidos pretérmino entre los años 2001-2002 en el Complejo Hospitalario Torrecárdenas de Almería; y, b) Grupo Control: 10 niños nacidos a término en la misma franja temporal en la provincia de Almería e igualados en nivel sociocultural, edad y sexo con los niños del grupo experimental (ver Tabla 1).

Los participantes del grupo control fueron seleccionados en los Colegios Públicos de Educación Infantil y Primaria (CEIPs) -José Díaz Díaz-, -Joaquín Visiedo- y -Lope de Vega- (Almería).

Tabla 1. Descripción de las características demográficas de la muestra.

\begin{tabular}{lll}
\hline Variables & Niños prematuros & Niños a término \\
\hline Edad media & 7.5 & 7.5 \\
Sexo & Femenino (6) & Femenino (6) \\
& Masculino (4) & Masculino (4) \\
& & Estudios Primarios (3) \\
Nivel sociocultural & Estudios Primarios (3) & Educación Secundaria (3) \\
& Educación Secundaria (3) & Estudios Universitarios (4) \\
\hline
\end{tabular}

A partir de los registros de prematuridad del Hospital Torrecárdenas de Almería, se realizó la selección de los niños nacidos pretérmino en base a: (i) Edad gestacional $\leq 32$ semanas y peso inferior a 1500 gr., y (ii) Inexistencia de patología neurológica conocida.

Quedaron excluidos de este estudio aquellos niños que presentaron antecedentes de traumatismo craneoencefálico, parálisis cerebral u otro diagnóstico neurológico, o déficits motores y/o sensoriales que impidiesen la evaluación neuropsicológica.

Todos los participantes incluidos en la muestra eran hispano hablantes. Los padres, o tutores legales de los niños, firmaron un consentimiento informado para que éstos pudiesen participar en el estudio.

\section{Instrumentos}

Historias Clínicas y Entrevistas. Para que la selección de la muestra de niños/as fuese lo más homogénea posible, con las historias clínicas y las entrevistas individualizadas se obtuvo información relativa a: edad gestacional, peso al nacer en gramos, fecha de nacimiento, sexo, edad y nivel sociocultural.

Posteriormente se evaluaron diversos dominios cognitivos - funciones ejecutivas, integración viso-perceptiva, coordinación visomotora, memoria, conocimiento del medio, lenguaje, procesamiento visomotor y aptitud motora/psicomotricidad fina (ver Tabla 2)-, elaborándose para tal fin un protocolo que incluía las siguientes pruebas neuropsicológicas estandarizadas. 
Tabla 2. Procesos cognitivos y pruebas neuropsicológicas.

\begin{tabular}{|c|c|}
\hline Procesos Cognitivos & Pruebas Neuropsicológicas \\
\hline \multicolumn{2}{|l|}{ Funciones ejecutivas: } \\
\hline \multirow[t]{4}{*}{ - Memoria de trabajo } & - Letras y Números (WISC-IV) \\
\hline & - Repetición de Números (B. Kaufman) \\
\hline & - Orden de Palabra (B. Kaufman) \\
\hline & - Dígitos Inversos (WISC-IV) \\
\hline - Atención dividida/Flexibilidad cognitiva & Trail Making B \\
\hline - Inhibición de respuestas/Supresión de interferencias & Stroop \\
\hline \multirow{2}{*}{ - Generación de estrategias/Flexibilidad cognitiva } & - Matrices Análogas (B. Kaufman) \\
\hline & - Triángulos (B. Kaufman) \\
\hline - Planificación/Organización/Decisión estratégica & Series de Fotos (B. Kaufman) \\
\hline - Atención sostenida/Vigilancia/Monitorización & Test de la "A" \\
\hline \multirow[t]{2}{*}{ - Atención selectiva } & Trail Making A \\
\hline & - Animales (AnA, AnO) (WISC-IV) \\
\hline - Iniciativa/Razonamiento conceptual/Planificación/Estrategia & - Aritmética (B. Kaufman) \\
\hline - Fluencia verbal fonológica & $\checkmark$ FAS \\
\hline - Fluencia verbal semántica & Animales \\
\hline \multirow{2}{*}{ - Velocidad de procesamiento/Eficacia } & - Búsqueda de Símbolos (WISC-IV) \\
\hline & - Animales (AnA, AnO) (WISC-IV) \\
\hline \multirow[t]{3}{*}{ Integración viso-perceptiva: } & - Cierre Gestáltico (B. Kaufman) \\
\hline & - Triángulos (B. Kaufman) \\
\hline & - Figura de Rey (copia) \\
\hline Coordinación viso-motora: & - Movimiento de Manos (B. Kaufman) \\
\hline \multicolumn{2}{|l|}{ Memoria: } \\
\hline \multirow[t]{3}{*}{ - A corto plazo (ACP) } & - Recuerdo de la Figura de Rey a los 5’ \\
\hline & - Movimiento de Manos (B. Kaufman) \\
\hline & - Memoria Espacial (B. Kaufman) \\
\hline - A largo plazo (ALP) & - Recuerdo de la Figura de Rey a los 15' \\
\hline Conocimiento del medio: & Caras y Lugares (B. Kaufman) \\
\hline \multirow[t]{4}{*}{ Lenguaje: } & - Test de Vocabulario en Imágenes Peabody (TVIP) \\
\hline & - Lectura/Decodificación (B. Kaufman) \\
\hline & - Lectura/Comprensión (B. Kaufman) \\
\hline & - Adivinanzas (B. Kaufman) \\
\hline \multirow[t]{4}{*}{ Procesamiento viso-motor: } & - Tiempo de Copia de la Figura de Rey \\
\hline & - Tiempo Trail Making (A/B) \\
\hline & - Tiempo Triángulos \\
\hline & - Tiempo Series de fotos \\
\hline Aptitud motora y psicomotricidad fina: & - Dibujo de un niño/a (Escalas McCarthy) \\
\hline
\end{tabular}

Adaptación Hispanoamericana del Test de Vocabulario en Imágenes Peabody (TVIP; Dunn, Padilla, Lugo y Dunn, 1986), a fin de conocer el nivel premórbido de los niños y obtener un índice de su edad mental. Los estudios de validez han demostrado que correlaciona bien con otros tests de vocabulario y tests individuales de inteligencia, especialmente con las escalas Wechsler y Stanford-Binet (Robertson y Eisenberg, 1981).

Adaptación Española de la Batería de Evaluación de Kaufman para niños (K-ABC; Conde Torrijos, 1997). En el manual de la prueba se citan 43 estudios de validez llevados a cabo antes de su publicación; las correlaciones con pruebas como la Escala de Inteligencia de Wechsler (WISC) y la Stanford-Binet fueron las más replicadas (Bloom et al., 1988; Hendershott, Searight, Russell, Hatfield y Rogers, 1990; Naglieri y Anderson, 1985). Se administraron los Test de la Escala de Procesamiento Secuencial, compuestos por problemas que deben ser resueltos partiendo de secuencias o series ordenadas, ha- bilidad íntimamente unida a una gran variedad de tareas escolares:

- Movimiento de Manos (MM), requiere capacidad de organización perceptiva, reproducción de modelos, memoria a corto plazo, habilidad espacial y, muy especialmente, coordinación visomotora.

- Repetición de Números (RN), mide atención y memoria de trabajo.

- Orden de Palabra (OP), evalúa capacidad de memoria de trabajo, con y sin interferencias de otros estímulos.

De la Escala de Procesamiento Simultáneo, que requiere que la información sea integrada y sintetizada simultáneamente para producir la solución adecuada, se aplicaron los siguientes test:

- Cierre Gestáltico (CG), supone habilidades de integración viso-perceptiva -cierre perceptivo, interferencia perceptiva y conversión del estímulo abstracto en un objeto 
concreto-, flexibilidad en la percepción y en el pensamiento.

- Triángulos (TRIANG), implica integración visoperceptiva, habilidades de orientación y representación espacial, generación de estrategias de análisis del todo y las partes y flexibilidad cognitiva.

- Matrices Análogas (MA), evalúa la capacidad de generar sistemáticamente estrategias de respuesta ante ítems abstractos. Se requiere flexibilidad cognitiva y capacidad de razonamiento abstracto.

- Memoria Espacial (ME), requiere concentración y memoria a corto plazo.

- Series de Fotos (SF), mide capacidad de seriación, relaciones temporales y sentido común de la relación causa efecto. Se requieren habilidades de planificación, organización y decisión estratégica.

De la Escala de Conocimientos, que proporciona una medida del nivel de información, vocabulario expresivo, relaciones y estructuración del lenguaje, conceptos aritméticos, decodificación lectora y lectura comprensiva, es decir, conocimientos estrechamente vinculados a hechos y/o habilidades adquiridas como consecuencia de la estimulación ambiental o de la escolaridad, se administraron los siguientes test:

- Caras y Lugares (CL), mide el nivel de formación del niño, partiendo de estímulos visuales que son personajes o lugares famosos. Ésta es la prueba con mayor influencia cultural, implicando un conocimiento del medio.

- Aritmética (ARIT), evalúa la habilidad del niño para identificar números, contar, resolver problemas de la vida diaria y demostrar comprensión de problemas matemáticos. Se requiere iniciativa, razonamiento conceptual, planificación y organización estratégica.

- Adivinanzas (ADIV), proporciona una medida de destrezas normalmente asociadas con el conocimiento general y la inteligencia verbal (lenguaje). Esto es, evalúa la habilidad del niño para deducir el nombre de un concepto verbal concreto o abstracto, cuando se le proporcionan varias de sus características.

- Lectura Decodificación (LD), mide la habilidad para identificar letras, sílabas y palabras, pronunciándolas correctamente (lenguaje).

- Lectura Comprensión (LC), evalúa la comprensión lectora a través de gestos o respuestas motrices.

Adaptación Española de la Escala de Inteligencia de Weschlerpara niños-IV (WISC-IV; Corral, Arribas, Santamaría, Sueiro y Pereña, 2005) con una validez de contenido excelente (5) y una validez de constructo adecuada (3). Del índice de Memoria de Trabajo (MT), que mide la capacidad para retener y almacenar información, operar mentalmente con ella, transformarla y generar nueva información, se administraron las siguientes subescalas:

- Letras y Números (LN), analiza la capacidad de retener y combinar dos tipos de información, ordenarla y elaborar un conjunto organizado según consignas (memoria de trabajo).

- Dígitos en Orden Inverso (DI), evalúa memoria de trabajo, indicando habilidades de secuenciación, planificación, alerta y flexibilidad cognitiva.

Del índice de Velocidad de Procesamiento de la Información (VP), que mide la capacidad para focalizar la atención, explorar, ordenar y/o discriminar información visual con rapidez y eficacia, se administraron las siguientes subescalas, que se desarrollan bajo control de tiempo:

- Búsqueda de Símbolos (BS), mide habilidades de rapidez asociativa, aprendizaje, percepción visual, coordinación viso-motora, atención, motivación y resistencia frente a tareas repetitivas.

- Animales (ANIM), evalúa atención selectiva y planificación en la búsqueda ordenada versus desordenada de información.

Trail Making Test (TMT; Peña-Casanova et al., 2009; Strauss, Sherman y Spreen, 2006). Los coeficientes de validez son generalmente aceptables (ver Lezak, 1995, para una revisión).

- Parte $A$, mide habilidades motoras, viso-espaciales de búsqueda visual y atención selectiva -capacidad de establecer y mantener la respuesta-.

- Parte B, implica además de las habilidades mencionadas en la parte A, flexibilidad mental y atención dividida capacidad de inhibir una respuesta previamente aprendida-.

Test de la A (Strub y Black, 1985), evalúa la atención sostenida o vigilancia, y permite la detección de errores de omisión, perseveración y/o confusión.

Test Stroop (Martín et al., 2012; Peña-Casanova et al., 2009; Stroop, 1935), para la medida de la inhibición de respuestas.

Figura Compleja de Rey (Rey, 1980). La copia de esta figura permite explorar habilidades viso-perceptivas, viso-espaciales y viso-constructivas.

Respecto a la valoración de las distintas funciones de la memoria, los instrumentos utilizados fueron:

- Recuerdo de la Figura de Rey a los 5', explora la memoria visual a corto plazo.

- Recuerdo de la Figura de Rey a los 15', evalúa memoria visual a largo plazo.

Prueba de Fluencia Fonológica (FAS) y Semántica (Animales). Permiten valorar las funciones frontales (Strauss et al., 2006; Tombaught, Kozak y Rees, 1999). En la primera prueba se pidió la evocación de palabras que comenzasen por las letras F, A y S durante un minuto. En el segundo caso, los participantes debían evocar nombres de animales durante el mismo espacio de tiempo.

Finalmente, para evaluar el procesamiento visomotor se registraron los tiempos de respuesta en los siguientes tests: 
- Test de Copia de la Figura Compleja de Rey (Rey, 1980).

- Trail Making Test A/B (Peña-Casanova et al., 2009; Strauss et al., 2006).

- Triángulos. Escala de Procesamiento Simultáneo de la Adaptación Española de la Batería de Evaluación de Kaufman para niños (K-ABC; Conde Torrijos, 1997).

- Series de Fotos. Escala de Procesamiento Simultáneo de la Adaptación Española de la Batería de Evaluación de Kaufman para niños (K-ABC; Conde Torrijos, 1997).

Adaptación Española de las Escalas McCarthy de Aptitudes y Psicomotricidad para niños (MSCA; McCarthy, 2006). Se administró la subescala "Dibujo de un niño", que evalúa la aptitud motora y la psicomotricidad fina.

Finalmente, para agradecer su participación, al terminar la evaluación los niños podían elegir dos de los siguientes premios: bolígrafos, libretas, cromos, pegatinas y golosinas.

\section{Procedimiento}

En el caso del grupo de niños prematuros, éstos fueron seleccionados en base a los criterios anteriormente citados. Dicha selección se realizó a través de la revisión de las historias clínicas pertenecientes a todos los niños nacidos pretérmino entre los años 2001-2002 en el Complejo Hospitalario Torrecárdenas de Almería. Tomados los datos clínicos, se envió una carta certificada a cada padre proporcionándoles toda la información acerca de la investigación que se iba a llevar a cabo, solicitando su cooperación y la participación de su hijo/a, a través del formato oficial de consentimiento informado del Servicio Andaluz de Salud. Posteriormente, se contactó vía telefónica con cada padre, volviéndoles a dar información sobre la investigación y duración de la evaluación -ésta se realizaba en una sola sesión de tres horas, aproximadamente, incluidos los descansos que, como mínimo, fue- ron dos-, y se resolvían sus dudas concertando, finalmente, una cita con ellos y su hijo.

Una vez que aceptaron, fueron citados en diferentes días para proceder a su evaluación. Antes de realizarla, se aclararon nuevamente posibles dudas y se llevó a cabo una breve entrevista en la que se recogió información sobre las variables socio-demográficas y las características físicas del niño (se les preguntaba también sobre posibles alergias).

La evaluación se llevó a cabo en las consultas externas de pediatría del C. H. "Torrecárdenas", en horario de tarde y según disponibilidad de los padres. Dos fueron las personas encargadas de realizarla; mientras una administraba la batería de pruebas neuropsicológicas, otra registraba las respuestas $y$, cuando era necesario, los tiempos de ejecución con ayuda de un cronómetro.

En el caso del grupo control, primero se contactó con el equipo directivo de los CEIPs seleccionados y, a continuación, con las asociaciones de padres de dichos colegios. Se les explicó la investigación que se iba a realizar y se solicitó su cooperación. Una vez que accedieron a participar, se contactó con los profesores de las clases que iban a colaborar en el estudio y se acordaron los días para acudir al aula. Los niños fueron llamados de uno en uno, realizándose la evaluación en una sala facilitada por el centro. Tras finalizar, los niños regresaban a su aula con total normalidad.

\section{Resultados}

Los análisis de los datos obtenidos en todas las pruebas (ver Tabla 3) se realizaron mediante un Análisis de Varianza para un diseño unifactorial con un factor manipulado entregrupos, Grupo de pertenencia (prematuros vs. controles). Para ello se utilizó el programa estadístico SPSS v. 15.0 para Windows, estableciéndose el nivel de significación en $p \leq .05$.

Tabla 3. Media; Error Típico (entre paréntesis); Significación $\left({ }^{*} p \leq .05 ; * * p \leq .01 ; * * t \leq .001\right.$; n.s "no significativo").

\begin{tabular}{|c|c|c|c|}
\hline PRUEBAS & PREMATUROS & CONTROLES & SIGNIFICACIÓN \\
\hline \multicolumn{4}{|l|}{ Funciones ejecutivas } \\
\hline Letras y Números & $8.20(1.61)$ & $12.50(1.24)$ & $*$ \\
\hline Repetición de Números & $8.60(.63)$ & $10.60(.81)$ & n.s. \\
\hline Orden de Palabra & $9.70(.93)$ & $11.50(.77)$ & n.s. \\
\hline Dígitos Inversos & $3.50(.42)$ & $4.10(.36)$ & n.s. \\
\hline Trail Making B & $0.60(.16)$ & $1.00(.00)$ & $*$ \\
\hline Stroop & $46.10(5.16)$ & $51.20(1.57)$ & n.s. \\
\hline Trail Making A & $0.80(.13)$ & $1.00(.00)$ & n.s. \\
\hline Matrices Análogas & $11.00(.97)$ & $14.60(1.08)$ & $*$ \\
\hline Series de Fotos & $7.30(1.09)$ & $13.60(.84)$ & $* * *$ \\
\hline Test de la "A" & $0.60(.16)$ & $0.90(.10)$ & n.s. \\
\hline Aritmética & $92.60(3.71)$ & $107.90(3.42)$ & $* *$ \\
\hline FAS & $17.30(2.32)$ & $25.60(2.69)$ & $*$ \\
\hline $\mathrm{F}$ & $5.00(.59)$ & $7.30(.61)$ & * \\
\hline A & $5.80(1.06)$ & $9.50(1.40)$ & $*$ \\
\hline S & $6.50(1.15)$ & $8.80(1.06)$ & n.s. \\
\hline Animales & $13.40(2.03)$ & $17.20(1.21)$ & n.s. \\
\hline Dibujo de un niño & $14.60(.56)$ & $15.80(.59)$ & n.s. \\
\hline
\end{tabular}




\begin{tabular}{|c|c|c|c|}
\hline PRUEBAS & PREMATUROS & CONTROLES & SIGNIFICACIÓN \\
\hline \multicolumn{4}{|l|}{ Integración viso-perceptiva } \\
\hline Cierre Gestáltico & $9.40(1.02)$ & $10.70(.71)$ & n.s. \\
\hline Triángulos & $10.30(1.33)$ & $14.10(.73)$ & $*$ \\
\hline Figura de Rey (copia) & $18.15(3.20)$ & $21.40(2.23)$ & n.s. \\
\hline \multicolumn{4}{|l|}{ Coordinación viso-motora } \\
\hline Movimiento de Manos & $11.20(.86)$ & $14.00(1.09)$ & n.s. \\
\hline \multicolumn{4}{|l|}{ Velocidad de procesamiento } \\
\hline Búsqueda de Símbolos & $11.40(.86)$ & $13.60(.52)$ & * \\
\hline Animales & $7.60(1.04)$ & $0.20(.78)$ & n.s. \\
\hline AnA & $7.20(.92)$ & $10.40(.58)$ & $* *$ \\
\hline $\mathrm{AnO}$ & $8.40(.96)$ & $10.10(1.01)$ & n.s. \\
\hline \multicolumn{4}{|l|}{ Memoria } \\
\hline Recuerdo F. Rey 5’ & $5.35(1.15)$ & $9.60(1.02)$ & $*$ \\
\hline Recuerdo F. Rey 15' & $6.45(1.12)$ & $9.75(1.37)$ & n.s. \\
\hline Memoria Espacial & $10.60(1.20)$ & $11.50(1.10)$ & n.s. \\
\hline \multicolumn{4}{|l|}{ Conocimiento del medio } \\
\hline Caras y Lugares & $94.60(3.95)$ & $115.10(4.79)$ & $* *$ \\
\hline \multicolumn{4}{|l|}{ Lenguaje } \\
\hline Lenguaje/Decodificación & $123.00(7.93)$ & $137.90(.67)$ & n.s. \\
\hline Lenguaje/Comprensión & $193.20(89.54)$ & $101.50(10.19)$ & n.s. \\
\hline Adivinanzas & $98.50(3.93)$ & $118.50(4.09)$ & $* *$ \\
\hline \multicolumn{4}{|l|}{ Procesamiento viso-motor } \\
\hline Tiempo Trail Making A & $131.40(14.73)$ & $108.95(9.48)$ & $*$ \\
\hline Tiempo Trail Making B & $234.60(14.86)$ & $175.90(12.31)$ & $* *$ \\
\hline Tiempo Triángulos & $52.66(3.17)$ & $42.36(2.44)$ & $*$ \\
\hline Tiempo Serie de Fotos & $22.64(2.20)$ & $32.47(3.48)$ & $*$ \\
\hline Tiempo Copia F. de Rey & $359.90(36.28)$ & $255.40(23.80)$ & $*$ \\
\hline Tiempo Recuerdo 5’ F. de Rey & $140.20(33.70)$ & $105.80(14.18)$ & n.s. \\
\hline Tiempo Recuerdo 15’ F. de Rey & $95.80(14.31)$ & $86.80(11.83)$ & n.s. \\
\hline
\end{tabular}

Los resultados mostraron diferencias estadísticamente significativas en las siguientes pruebas: Peabody $(F(1,18)=$ $6.900, p<.05)$; Letras y Números $(F(1,18)=4.448, p<.05)$; Trail Making $(\mathrm{B})(F(1,18)=6.000, p<.05)$; Matrices Análogas $(F(1,18)=6.062, p<.05)$; Series de Fotos $(F(1,18)=20.708$, $p<.001)$; Aritmética $(F(1,18)=9.171, p<.01)$; FAS $(F(1,18)=$ $5.436, p<.05) ; \mathrm{F}(F(1,18)=7.203, p<.05) ; \mathrm{A}(F(1,18)=$ 4.399, $p<.05)$; Triángulos $(F(1,18)=6.218, p<.05)$; Búsqueda de Símbolos $(F(1,18)=4.694, p<.05) ; \operatorname{AnA}(F(1,18)=8.533$, $p<.01)$; Recuerdo Rey 5' $(F(1,18)=7.635, p<.05)$; Caras $y$ Lugares $(F(1,18)=10.854, p<.01)$; Adivinanzas $(F(1,18)=$ $12.418, p<.01)$; Tiempo Trail A $(F(1,18)=7.538, p<.05)$; Tiempo Trail B $(F(1,18)=9.248, p<.01)$; Tiempo Triángulos $(F(1,18)=6.606, p<.05)$; Tiempo Series de Fotos $(F(1,18)=$ $5.694, p<.05)$; y Tiempo Copia Figura de Rey $(F(1,18)=$ $5.798, p<.05)$. Por tanto, tras el análisis de las medias fue posible verificar que los niños prematuros rendían significativamente menos que el grupo de niños controles. En el resto de pruebas que evaluaban inhibición de respuestas, atención sostenida, atención selectiva, fluencia verbal semántica, coordinación viso-motora, memoria a largo plazo, aptitud motora y psicomotricidad fina, ambos grupos mostraron una ejecución similar ( $p>$.05).

\section{Discusión}

El principal objetivo de este trabajo fue realizar una evaluación de las posibles secuelas neuropsicológicas a los 7 años de edad en niños nacidos pretérmino, en comparación con los nacidos a término. Los resultados muestran que los niños prematuros presentan alteraciones centradas en las funciones ejecutivas, la integración viso-perceptiva, la memoria a corto plazo, el conocimiento del medio y el lenguaje. Asimismo, el índice -edad mental- obtenido mediante el Test de Vocabulario en Imágenes Peabody (TVIP), es significativamente inferior en los niños prematuros que el que cabría esperar según su edad cronológica.

En la evaluación neuropsicológica se observaron diferencias entre ambos grupos en funciones ejecutivas tales como el establecimiento de conductas dirigidas a meta iniciativa, razonamiento conceptual, toma de decisiones, planificación y generación de estrategias para la resolución de problemas-. También se encontraron déficits en memoria de trabajo, atención dividida, flexibilidad cognitiva y fluencia fonológica. Estos resultados están en consonancia con los trabajos de Elliot (2003), Korzeniowski (2011), Lezak (2004) y Verdejo-García y Bechara (2010), que resaltan el papel esencial que las funciones ejecutivas tienen en la regulación 
de una conducta orientada a una meta. O los estudios de Aarnoudsse-Moens et al. (2009) en los que se muestra que las principales áreas neuropsicológicas alteradas en función ejecutiva en niños nacidos pretérmino son la memoria de trabajo, la flexibilidad cognitiva y la fluidez verbal -les resulta más difícil generar nombres pertenecientes a diferentes categorías (cambio de set mental) y presentan perseverancia en sus respuestas-.

También se encontraron diferencias en la tarea de integración viso-perceptiva "Triángulos", que implica no sólo habilidades de orientación y representación espacial sino, también, la generación de estrategias de análisis del todo y las partes así como flexibilidad cognitiva. Este resultado concuerda con investigaciones previas como las realizadas por Geldof et al. (2012), Litt et al. (2005), Olsén et al. (1998), Pasman et al. (1998) y Strang-Karlsson et al. (2010), quienes señalan que los niños prematuros presentan dificultades en los procesamientos viso-espaciales y viso-perceptivos (tareas de integración funcional), en comparación con los niños nacidos a término.

Por otra parte, se observó una ralentización del procesamiento mental, sobre todo en tareas que exigen control atencional, concentración, planificación y resistencia a la distracción (Búsqueda de símbolos, Animales -versión AnA-). Además, los tiempos de ejecución registrados en todas las pruebas de procesamiento visomotor -a excepción de Recuerdo a los 5' y a los 15'de la Figura de Rey-, fueron significativamente superiores a los obtenidos por los niños nacidos a término.

Un hallazgo interesante es el relativo al recuerdo a corto plazo de la Figura de Rey. A los cinco minutos, los niños nacidos pretérmino recuerdan menos detalles y suelen dibujar los elementos de manera aislada, sin existir un armazón general. No obstante, cuando se les pide a los 15 minutos que dibujen la figura, su recuerdo es equiparable al de los niños nacidos a término, aunque con menos detalles.

Finalmente, el grupo de niños prematuros presenta alteraciones en la resolución y adquisición de conocimientos del medio (Caras y Lugares), encontrándose también afectadas las habilidades verbales relacionadas con la comprensión auditiva de enunciados que enmascaran el nombre de un objeto o acción (Adivinanzas). Dichas alteraciones también aparecen reflejadas en los trabajos de Briscoe et al. (2001), Ment et al. (2003) y Peterson et al. (2002), quienes observaron dificultades en tareas que requerían habilidades verbales, comprensión gramatical y comprensión auditiva de nombres de dibujos.

\section{Referencias}

Aarnoudse-Moens, C. S., Weisglas-Kuperus, N., van Goudoever, J. B. y Oosterlaan, J. (2009). Meta-analysis of neurobehavioral outcomes in very preterm and/or very low birth weight children. Pediatrics, 124, 717728. doi: $10.1542 /$ peds.2008-2816

Anderson, P. (2002). Assessment and development of executive function (EF) during childhood. Child Neuropsychology, 8(2), 71-82. Recuperado de http://www.ncbi.nlm.nih.gov/pubmed/12638061
En el presente estudio no se han encontrado diferencias entre ambos grupos en dos dominios como son la coordinación viso-motora y la aptitud motora y/o psicomotricidad fina, lo que puede deberse a que dichas funciones son muy básicas y, por tanto, no se ven afectadas por la prematuridad.

\section{Conclusiones}

Debido al escaso número de trabajos que analizan procesos cognitivos en niños nacidos pretérmino de habla hispana en los primeros años de la infancia (6-11 años), en esta investigación se seleccionó la ventana de edad de 7 años, momento en el que se inician procesos esenciales como el aprendizaje de la lectura, la escritura y el cálculo matemático, áreas de especial relevancia, para detectar problemas neuropsicológicos que pudiesen estar influyendo, entre otras cosas, en el rendimiento académico de estos niños.

En este sentido, los resultados obtenidos muestran que los niños de siete años nacidos pretérmino presentan alteraciones neuropsicológicas en diversos dominios cognitivos que abarcan funciones ejecutivas, integración visoperceptiva, memoria a corto plazo, conocimiento del medio, lenguaje y procesamiento visomotor, demostrando que estos déficits pueden estar a la base de los problemas conductuales y de escolarización que esta población presenta o puede presentar en el futuro, lo que proporciona nuevos hallazgos en el ámbito de la neuropsicología infantil.

Conocer cuáles son es un primer paso de vital importancia para el posterior diseño de programas de entrenamiento específicos que sean eficaces para paliar o superar dichas dificultades.

\section{Limitaciones}

En este estudio se han evaluado diferentes procesos cognitivos en un grupo de niños nacidos pretérmino, utilizando pruebas neuropsicológicas estandarizadas. Sin embargo, no se ha explorado la relación de los resultados obtenidos en las mismas con el rendimiento académico o con su desempeño en tareas cotidianas. Para completarlo, sería conveniente en futuras investigaciones evaluar dicha relación, permitiendo de este modo predecir en qué grado los posibles déficits pueden condicionar el funcionamiento diario de estos niños en contextos significativos, como el hogar, el centro escolar $\mathrm{u}$ otros ambientes de ocio.

Anderson, P. J. (2008). Towards a developmental model of executive function. In V. Anderson, R. Jacobs, \& P. J. Anderson (Eds.), Executive functions and the frontal lobes: A lifespan perspective (pp. 3-22). New York: Psychology Press.

Bloom, A., Allard, A., Zelko, F., Brill, W., Topinka, C. y Pfohl, W. (1988). Differential validity of the K-ABC for lower functioning preschool 
children versus those of higher ability. American Journal of Mental Retardation, 93 (3), 273-277. doi: 10.1016/j.molcel.2011.09.016

Briscoe, J., Gathercole, S. E. y Marlow, N. (2001). Everyday memory and cognitive ability in children born very prematurely. Journal of child Psychology and Psychiatry, 42(6), 749-754. doi: 10.1111/1469-7610.00771

Burguet, A., Monnet, E., Roth, P., Him, F., Vouaillat, C., Lecour-Ducret, M., ... Menget, A. (2000). Devenir neurodéveloppemental à cinq ans des prématurés nés avant 33 semaines d'aménorrhée et indemnes d'infirmité motrice d'origine cérébrale. Archives de Pédiatrie, 7(4), 357-368. doi: 10.1016/S0929-693X(00)88830-2

Cassandra, B. y Reynolds, C. (2005). A Model of the Development of Frontal Lobe Functioning: findings from a Meta-Analysis. Applied Neuropsychology, 12(4), 190-201. doi: 10.1207/s15324826an1204_2

Corral, S., Arribas, D., Santamaría, P., Sueiro, M. J. y Pereña, J. (2005). Adaptación Española de la Escala de Inteligencia de Weschler para niños (WISC-IV). Departamento I+D de TEA Ediciones, S.A. Extraído el 31 de Marzo de 2007, de http://www.cop.es/uploads/PDF/WISCIV.pdf

Conde Torrijos, E. (1997). Adaptación Española de la Batería de Evaluación de Kaufman para niños $(K-A B C)$. TEA Ediciones, S.A.

Dunn, L. M., Padilla, E. R., Lugo, D. E. y Dunn, L. M. (1986). Adaptación Hispanoamericana del Test de Vocabulario en Imágenes Peabody (TVIP). Circle Pines, MN: American Guidance Service.

Elliott, R. (2003). Executive functions and their disorders. British Medical Bulletin; 65, 49-59. doi: 10.1093/bmb/65.1.49

Geldof, C. J. A., van Wassenaer, A. G., de Kieviet, J. F., Kok, J. H. y Oosterlaan, J. (2012). Visual perception and visual-motor integration in very preterm and/or very low birth weight children: A meta-analysis. Research in Developmental Disabilities, 33(2), 727-736. doi: 10.1016/j.ridd.2011.08.025

Hendershott, J., Searight, H., Russell, H., Hatfield, J. y Rogers, B. (1990). Correlations between the Stanford-Binet, Fourth Edition and the Kaufman Assessment Battery for Children for a preschool sample. Perceptual and Motor Skills, 71, 819-825.

Houd, O., Rossi, S., Lubin, A. y Joliot, M. (2010). Mapping numerical processing, reading, and executive functions in the developing brain: An fMRI meta-analysis of 52 studies including 842 children. Developmental Science, 13(6), 876-885. doi: 10. 1111/j.1467-7687.2009.00938.x

Isaacs, E. B., Edmonds, C. J., Lucas, A. y Gadian, D. G. (2001). Calculation difficulties in children of very low birthweight. Brain, 124, 1701-1707. doi: 10.1093/brain/124.9.1701

Johnson, M. H. (2000). Functional brain development in infants: elements of an interactive specialization framework. Child Development, 71, 75-81. doi:10.1111/1467-8624.00120

Johnson, S. P. (2003). The nature of cognitive development. Trends in Cognitive Science, 7, 102-104. doi: 10.1016/S1364-6613(03)00030-5

Karmiloff-Smith, A. (2007). Atypical epigenesist. Developmental Science, 10, 8488. doi: 10.1111/j.1467-7687.2007.00568.x

Korzeniowski, C. G. (2011). Desarrollo evolutivo del funcionamiento ejecutivo y su relación con el aprendizaje escolar. Revista de Psicología 7(13), 726. Extraído el 8 de Octubre de 2014, de http://bibliotecadigital.uca.edu.ar/repositorio/revistas/desarrolloevolutivo-funcionamiento-ejecutivo.pdf

Lezak, M. D. (1995). Neuropsychological Assessment $3^{\text {rd }}$ ed. New York: Oxford University Press.

Lezak, M. D. (2004). Neuropsychological assessment. New York: Oxford University Press.

Litt, J., Taylor, H. G., Klein, N. y Hack, M. (2005). Learning disabilities in children with very low birthweight: Prevalence, neuropsychological correlates, and educational interventions. Journal of Learning Disabilities, 8 , 130-141. doi: 10.1177/00222194050380020301

McCarthy, D. (2006). Adaptación Española de las Escalas McCarthy de Aptitudes y Psicomotricidad para niños (MSCA). Departamento I+D de TEA Ediciones, S.A.

Marlow, N., Wolke, D., Bracewell, M. A. y Samara, M. (2005). Neurologic and developmental disability at six years of age after extremely preterm birth. New England Journal of Medicine, 352, 9-19. doi: 10.1056/NEJMoa041367

Martín, R., Hernández, S., Rodríguez, C., García, E., Díaz, A. y Jiménez, J. E. (2012). Datos normativos para el Test de Stroop: patrón de desarro- llo de la inhibición y formas alternativas para su evaluación. European Journal of Education and Psychology, 5(1), 39-51. doi: 10.1989/ejep.v5il.89

Mathiasen, R., Hansen, B. M., Forman, J. L., Kessing, L. V. y Greisen, G. (2011). The risk of psychiatric disorders in individuals born prematurely in Denmark form 1974 to 1996. Acta Paediatrica, 100, 691-699. doi: 10.1111/j.1651-2227.2011.02136.x

Mellier, D. (2004). Les bébés nés prématurément: quels risques pour le développement psychologique? En R. Lécuyer (Eds.), Le développement du nourrison (pp. 309-346). Paris: Dunod.

Ment, L., Vohr, B., Allan, W., Katz, K. H., Schneider, K. C., Westerveld, M., ... Makuch, R. W. (2003). Change in cognitive function over time in very low birth weight infants. The Journal of the American Medical Association, 289(6), 705-712.

Naglieri, J. y Anderson, D. (1985). Comparison of the WISC-R and K-ABC with gifted students. Journal of Psychoeducational Assessment, 3, 175-179. doi: $10.1177 / 073428298500300208$

Narberhaus, A. y Segarra, D. (2004). Trastornos neuropsicológicos y del neurodesarollo en el prematuro. Anales de Psicología, 20, 317-326. doi:10.6018/27511

O’Brien, F., Roth, S., Stewart, A., Rifkin, L., Rushe, T. y Wyatt, J. (2004). The neurodevelopmental progress of infants less than 33 weeks into adolescence. Archives of Disease in Childhood, 89, 207-211. doi: 10.1136/adc.2002.006676

O'Hare, E. D., Lu, L. H., Houston, S. M., Bookheimer, S. Y. y Sowell, E. R. (2008). Neurodevelopmental changes in verbal working memory loaddependency: an fMRI investigation. Neuroimage, 42, 1678-1685. doi: 10.1016/j.neuroimage.2008.05.057

Olsén, P., Vainionpää, L., Pääkkö, E., Korkman, M., Pyhtinen, J. y Järvelin, M. R. (1998). Psychological findings in preterm children related to neurologic status and magnetic resonance imaging. Pediatrics 102(2), 329336. doi: 10.1542/peds.2011-1735

Pasman, J. W., Rottevel, J. J. y Maassen, B. (1998). Neurodevelopmental profile in low risk preterm infants at 5 years of age. European Journal of Paediatric Neurology, 2, 7-17. doi: 10.1016/1090-3798(98)01000-7

Peña-Casanova, J., Quiñones-Úbeda, S., Quintana-Aparicio, M., Aguilar, M., Badenes, D. y Molinuevo, J. L. (2009). Spanish Multicenter Normative Studies (NEURONORMA Project): Norms for Verbal Span, Visuospatial Span, Letter and Number Sequencing, Trail Making Test, and Symbol Digit Modalities Test. Archives of Clinical Neuropsychology, 24, 321-341. doi: 10.1093/archin/acp038

Peña-Casanova, J., Quiñones-Úbeda, S., Gramunt-Fombuena, N., Quintana, M., Aguilar, M., Molinuevo, J. L. y Serradell, M. (2009). NEURONORMA Study Team: Spanish Multicenter Normative Studies (NEURONORMA Project): Norms for the Stroop Color-Word Interference Test and the Tower of London-Drexel. Archives of Clinical Neuropsychology, 24, 413-429. doi: 10.1093/arclin/acp043

Peterson, B., Vohr, B., Staib, L., Cannistraci, C. J., Dolberg, A., Schneider, K. C., ... Ment, L. R. (2000). Regional brain volume abnormalities and long-term cognitive outcome in preterm infants. The Journal of the American Medical Association, 284(15), 1939-1947. doi: 10.1001/jama.284.15.1939

Peterson, B., Vohr, B., Kane, M. J., Whalen, D. H., Schneider, K. C., Katz, K. H., ... Ment, L. R. (2002). A functional magnetic resonance imaging study of language processing and its cognitive correlates in prematurely born children. Pediatrics, 110(6), 1153-1162. doi: 10.1542/peds.20052870

Portellano, J. A. (2005). Cómo desarrollar la inteligencia. Entrenamiento neuropsicológico de la atención y las funciones. Madrid, SP: Samos.

Rey, A. (1980). Test de copia de una figura compleja. 3 ed. revisada. Madrid: TEA Ediciones.

Robertson, J. R. y Eisenberg, J. L. (1981). Technical Supplement to the Peabody Picture Vocabulary Test-Revised. Circle Pines, MN, U.S.A.: American Guidance Service.

Salt, A. y Redshew, M. (2006). Neurodevelopmental follow-up after preterm birth: follow-up after two years. Early Human Development, 82, 185-197. doi: 10.1016/j.earlhumdev.2005.12.015

Strang-Karlsson, S., Andersson, S. y Paile-Hyvarinen, M. (2010). Slower reaction times and impaired learning in young adults with birth weight $<$ 1500 g. Pediatrics, 125, 74-85. doi: 10.1542/peds.2009-1297 
Strauss, E., Sherman, E. y Spreen, O. (2006). A compendium of neuropsychological tests. Administration, norms, and commentary. Oxford: University Press.

Stroop, J. R. (1935). Studies of interference in serial verbal reactions. Journal of Experimental Psychology, 18, 643-662. Extraído el 28 de Septiembre de 2014, de http://dx.doi.org/10.1037/h0054651

Strub, R. L. y Black, F. W. (1985). The Mental Status Examination in Neurology ( $4^{\text {th }}$ ed.). Philadelphia, F.A. Davis.

Stuss, D. T. y Knight, R. T. (2013). Principles of Frontal Lobe Function. New York: Oxford University Press.

Tombaught, T. N., Kozak, J. y Rees, L. (1999). Normative data stratified by age and education for two measures of verbal fluency: FAS and animal naming. Archives of Clinical Neuropsychology 14(2), 167-177. doi: $10.1093 / \operatorname{arclin} / 14.2 .167$
Verdejo-García, A. y Bechara, A. (2010). Neuropsychology of Executive Functions. Psicothema, 22(2), 227-235. Extraído el 8 de Octubre de 2014, de http://www.psicothema.com/pdf/3720.pdf

Wolosin, S. M., Richardson, M. E., Hennessey, J. G., Denckla, M. B. y Mostofsky, S. H. (2009). Abnormal cerebral cortex structure in children with ADHD. Human Brain Mapping, 30(1), 175-184. doi: 10.1002/hbm.20496

Zelazo, P. D., Müeller, U., Frye, D. y Marcovitch, S. (2003). The development of executive function in early childhood. Monographs of the Society for Reserch in Child Development, 6, Serial No. 274. doi: 10.1111/j.0037976X.2003.00266.x

(Artículo recibido: 30-04-2012; revisado: 01-06-2013; aceptado: 05-01-2015) 